\title{
Comparative Study of Interleaves in Communication System: IDMA Overview
}

\author{
Pradeepti Bisht ${ }^{1}$, Saurabh Mishra ${ }^{2}$ \\ ${ }^{1,2}$ (Electronics and Communication Department, DIT Dehradun, India)
}

\begin{abstract}
This paper present a review on the concept of Interleave Division Multiple Access (IDMA) which exploits the interleaving as only means of user separation. In this paper, different interleavers have been compared based on bit error rate. Finally it concludes few ideas on the future scope and challenging issues in this scheme.
\end{abstract}

Keywords: IDMA Receiver, Interleaving, Modulation Techniques, Spreading Codes, Tree Based Interleaver (TBI)

\section{Introduction}

In the recent years, the request for bandwidth has begun to surpass the availability in wireless networks. There are different multiple access techniques that have been studied to improve the bandwidth, efficiency and increase the number of users that can be accommodated within each cell [1]. These techniques used in 1G/2G/3G systems (such as FDMA/TDMA/CDMA respectively) were only suited for voice communication and unsuitable for high data rate transmission and burst data traffic. A $4 \mathrm{G}$ system is likely to provide a comprehensive and secure all possible solution where facilities such as IP telephony, ultra-broadband internet access, gaming services and streamed multimedia may be provide to users [1] [2]. The multiple access techniques which were nominated for 4G system were named as DS-CDMA (Direct Spread-Code Division Multiple Access), MC-CDMA (Multicarrier-Code Division Multiple Access), OFDMA (Orthogonal Frequency Division Multiple Access), IDMA (Interleave Division Multiple Access) etc.

\section{Concept Of Idma System}

It appears that Interleave Division Multiple Access have become an apparent as the dynamic candidate for the third generation wireless personal communication systems. IDMA inherits advantages of CDMA with one spreading sequence for all users, depend on interleaving to distinguish and separate signals from different users. Fig.1 below shows an IDMA system over a MAC. The interleaver/deinterleaver is the main component in the IDMA transmitter and receiver; though the choice of effective interleaver has a positive influence on the IDMA performances.At the transmitter for user $\mathrm{k}$, the information sequence for user $\mathrm{k}$ is initially encoded by an FEC encoder $\left(\mathrm{ENC}_{\mathrm{k}}\right)$ with rate $\mathrm{R}$ and then interleaved by an interleaver $\pi_{\mathrm{k}}$ into a chip sequence $\left\{\mathrm{x}_{\mathrm{k}}(\mathrm{j})\right\}$.

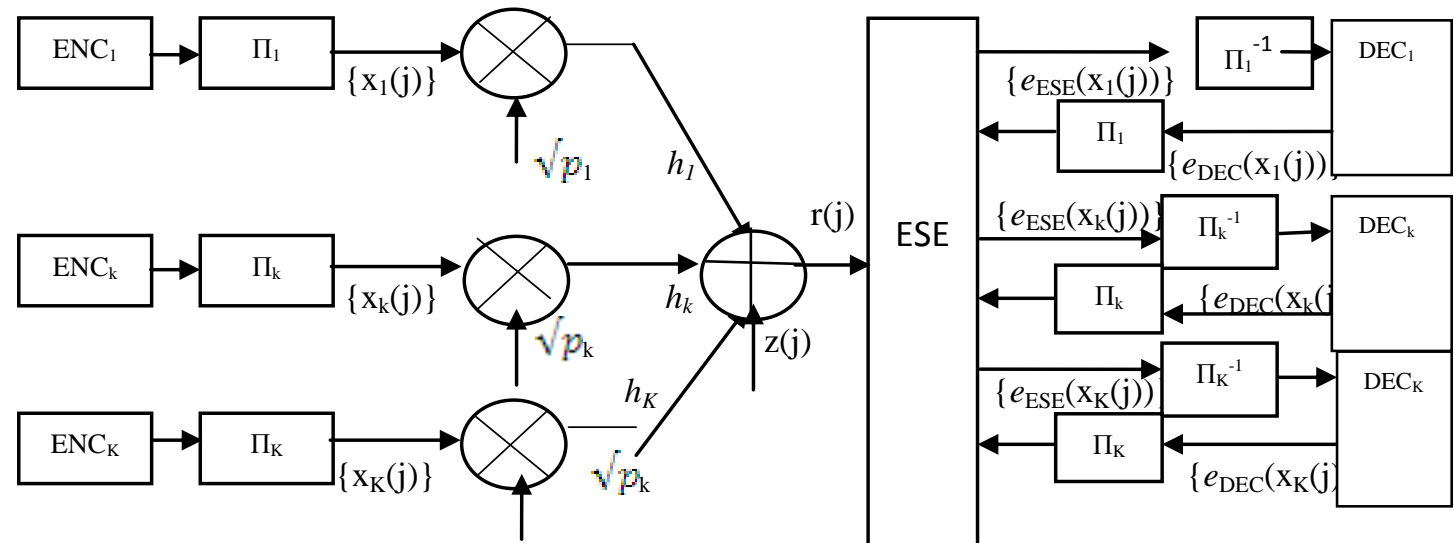

Figure 1. The system model of an IDMA multiple access scheme

A power control factor $\sqrt{p_{\mathrm{k}}}$ is used before transmission.

Initially, let us supposethere is perfect synchronization and no ISI. Assume that the channel is perfectly known at the receiver, then the received signal can be written as:

$r(j)=\sum_{k=1}^{K} \sqrt{ } p_{\mathrm{k}} h_{\mathrm{k}} x_{\mathrm{k}}(j)+z(j)$ 
Where $h_{\mathrm{k}}$ is the channel coefficient for user-k and $z(j)$ an AWGN with zero-mean and variance $\sigma^{2}=\mathrm{No} / 2$. The key to the low-cost detection strategy is the Gaussian approximation below [3][4]. Focusing on user- $k$ we rewrite (1) as:

$r(j)=\sqrt{ } p_{\mathrm{k}} h_{\mathrm{k}} x_{\mathrm{k}}(j)+\varepsilon_{\mathrm{k}}(j)$

Where $\varepsilon_{\mathrm{k}}(\mathrm{j})$ is the noise-plus-interference component in $r(j)$ in (1) with respect to $x_{\mathrm{k}}(j)$. Based on (2), the log-likelihood ratio (LLR) estimate of $x_{\mathrm{k}}(j)$ is calculated as: $e_{E S E}\left(x_{k}(j)\right)=\operatorname{Pr}\left(x_{k}(j)=+1\right)=2 \sqrt{p_{\mathrm{k}}} h_{\mathrm{k}}\left(r(j)-E\left(\varepsilon_{\mathrm{k}}(j)\right)\right)$

The evaluation in (3) is processed by repeatedly updating $E\left(\varepsilon_{\mathrm{k}}(j)\right)$ and $\operatorname{Var}\left(\varepsilon_{k}(j)\right)$ based on the feedback information $\left\{e_{\mathrm{DEC}}\left(x_{\mathrm{k}}(j)\right)\right\}$ from the decoders $\left\{\mathrm{DEC}_{\mathrm{k}}\right\}$ in Fig. $1[4]$

Recently, a semi-analytical tool, signal to noise-plus- interference ratio (SNIR) technique has been emerged to provide faster and more convincing performance evaluation for IDMA systems. Therefore, it is clearly seen that the above approach is MUD, not SUD, since the updating of $E\left(\varepsilon_{\mathrm{k}}(j)\right)$ and $\operatorname{Var}\left(\varepsilon_{k}(j)\right)$ in (3) involves feedback information from all users decoders [4].

\section{Analysis Of Interleavers In Idma}

Interleaving is a method of reshuffling the ordering of a data sequence in a one to one deterministic format. Interleaving is a technique for making forward error correction more durable with respect to burst errors. The basic role of an interleaver is to construct a long block code from small memory convolution codes, as long codes can approach the Shannon capacity limit. Moreover, it spreads out burst errors. The interleaver provides scrambled information data to the second component encoder and decorrelates inputs to the two component decoders so that an iterative suboptimum-decoding algorithm based on uncorrelated information exchange between the two component decoders can be applied. The final role of the interleaver is to break low weight input sequences, and thus increase the code free Hamming distance or reduce the number of code words with small distances is the code distance spectrum.

\subsection{Random Interleavers}

The role of random interleavers is to scramble the data of different users with different permutation pattern. Patterns of scrambling the data of users are generated arbitrarily. Because of the scrambling of data, burst error of the channel is randomized at the receiver side. The Random Interleaver block rearranges the elements of its input vector using a random permutation. But these interleavers demand a large amount of memory space at the transmitter and receiver ends for the purpose of their storage. Besides, considerable amount of bandwidth will be utilized for transmission of all these interleaver as well as computational complexity will be increased at receiver ends.

\subsection{Master Random Interleavers}

Later random interleavers were replaced by master random interleavers (MRI) so as to reduce the memory requirement raised due to storage of random interleavers at transmitter and receiver ends.Here in a master random interleaver or 'power-interleaver' method, a master interleaver pattern $\varphi$ is assigned, each user has a user specific interleaver $\left\{\pi_{\mathrm{k}}\right\}$ having length equal to chip length 'J'. Then $K(K$ is an integer) interleavers can be created using $\left\{\pi_{\mathrm{k}}=\varphi_{\mathrm{k}}\right\}$.

Here, $\varphi_{\mathrm{k}}(c)$ is,

$\varphi_{1}(c)=\varphi(c)$

$\varphi_{2}(c)=\varphi(\varphi(c))$

$\left.\varphi_{3}(c)=(\varphi(\varphi(c)))\right)$

By this procedure, every interleaver is a 'power' of $\varphi$.

The concept for this procedure is that if $\varphi$ is an 'ideal' random permutation, so are all $\left\{\varphi_{\mathrm{k}}\right\}$, and these permutations are also approximately independent from each other. Now BS assigns the power index $k$ to each user $k$, and then $\varphi_{\mathrm{k}}$ will be generated at the MS for user $k$ accordingly. This process of generating patterns increases the performance in the term information that has to be sending by the base station to the mobile station.

This method not only reduces the amount of information exchange between Base Station (BS) and Mobile Station (MS), but also considerably reduces the memory cost in comparison to random interleaver. 


\subsection{Tree Based Interleaver (TBI)}

In [5], M. Shukla et al proposed Tree Based Interleaver (TBI) to reduce the affect affiliated to bandwidth and memory requirements in addition to a matter of computational complexity optimally. In case of generation mechanism of tree based interleaver, two master interleavers are chosen, randomly. Let $\pi_{1}$ and $\pi_{2}$ be the two randomly chosen interleavers. The combinations of these two interleavers in a certain manner as shown in the Figure(2) are used as interleaving masks for the users [6] the allocations of the interleaving masks follow the binary tree format. The interleaving masking diagram is shown in Fig.2 for 14 users which may be upgraded for higher count of users. It is clearly shown through the Fig. 2 that, for obtaining the interleaving sequence related to $14^{\text {th }}$ user, it needs only 2 cycles.

$\pi_{14}=\pi_{2}\left(\pi_{2}\left(\pi_{2}\right)\right)$.
$\pi_{7}=\pi_{1}\left(\pi_{1}\left(\pi_{1}\right)\right)$.
$\pi_{13}=\pi_{1}\left(\pi_{2}\left(\pi_{2}\right)\right)$.

The algorithm for generation of TBI tree is given below.

Step 1: Master interleaver $\pi_{1}$ is randomly generated having the length of data block (data length $*$ spreader length).

Step 2: Master interleaver $\pi_{2}$ is randomly generated having the length of data block (data length $*$ spreader length).

Step 3: According to user k, level (L) of Tree is determined.

Hence, Number of users in that level $=2^{\mathrm{L}}$

Step 4: All the possible combinations $\left(2^{\mathrm{L}}\right)$ of interleavers are generated as $\pi_{1}\left(\pi_{2}\right), \pi_{2}\left(\pi_{1}\right) \ldots \ldots \ldots$.and so on.

Step 5: According to the user $\mathrm{k}$ particular combination of Master interleaver i.e. $\pi_{1}$ and $\pi_{2}$, is chosen and there after data is interleaved accordingly.

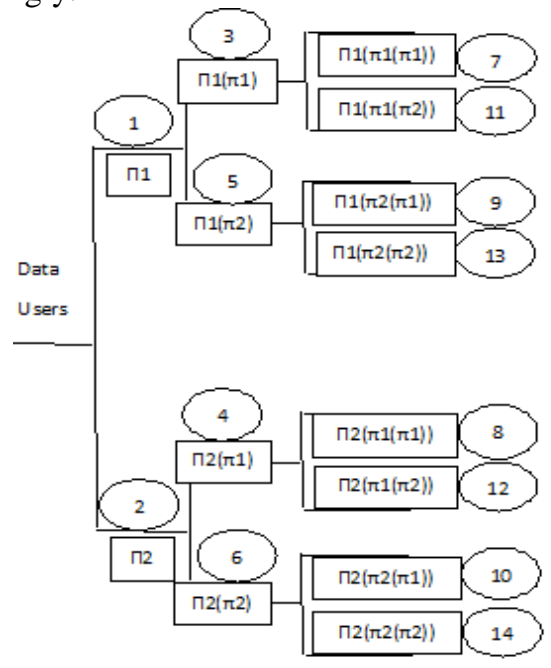

Figure 2. Interleaving Figure mask allocation for the proposed Tree Based Interleaving scheme

\subsection{Prime Interleaver}

Prime interleaver is based on prime number which gives a novel user-specific interleaver generation mechanism with lesser time to get it generated and along with minimal consumption of bandwidth required during transmission well similar performance in terms of BER to that of random interleaver. The Prime Interleaver is fundamentally aimed to reduce the bandwidth and memory requirement during transmission with lesser time, that occur in other available interleaver with BER performance comparable to random interleaver.

Now, user-specific seeds are assigned to different users. For understanding the mechanism of prime interleaver, let us examine a case of interleaving $n$ bits with seed p. Firstly, we observe a Galois field GF (n) and then the bits are interleaved with a distance of seed over GF (n) [Shukla 2010].

In case, if $\{1,2,3,4,5,6,7,8 \ldots \ldots \ldots \ldots n\}$ are consecutive bits to be interleaved with seed $p$ then location of bits after interleaving will be as follows:

$1===>1$

$2===>(1+p) \bmod n$

$3===>(1+2 \mathrm{p}) \bmod \mathrm{n}$ 


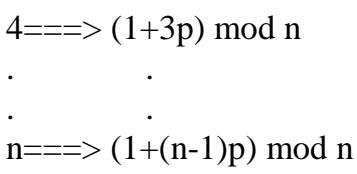

For example if we have to interleave 8 bits such that $\{1,2,3,4,5,6,7,8\}$ and we wish to interleave these bits with seed 3 then the new location of bit will be as follows

$$
\begin{aligned}
& 1===>1 \\
& 2===>(1+1 * 3) \bmod 3===>4 \\
& 3===>(1+2 * 3) \bmod 3===>7 \\
& 4===>(1+3 * 3) \bmod 3===>2 \\
& 5===>(1+4 * 3) \bmod 3===>5 \\
& 6===>(1+5 * 3) \bmod 3===>8 \\
& 7===>(1+6 * 3) \bmod 3===>3 \\
& 8===>(1+7 * 3) \bmod 3===>6
\end{aligned}
$$

Now, the new order of bits will be $\{1,4,7,2,5,8,31$ and 6$\}$.

Prime Interleavers require less bandwidth as compared to other available interleavers as now only seed is to be transmitted. In addition to this a very small amount of memory is required at the transmitter and receiver side. Thus this scheme reduces the computational complexity that occurs in random interleaving scheme.

\subsection{Two Dimensional Prime Interleavers}

In this type of interleaver, the aim of extending from 1D prime interleaver [7] into two dimensional is employed. Consider the case of two-dimensional interleaving of $n_{\mathrm{r}}$ by $n_{\mathrm{c}}$ matrix. Firstly, we split the interleaving scheme into row-wise interleaving and column-wise interleaving. Secondly, we assign the value of seed as row-wise seed and column-wise seed to row-wise and column-wise interleavers respectively. Therefore, the location of bits after interleaving will be as follows:

$$
\begin{array}{lc}
\text { Row-wise } & \text { Column-wise } \\
1===>1 & 1==>1 \\
2===>\left(1+p_{\text {row }}\right) \bmod n_{\mathrm{r}} & 2==>\left(1+p_{\text {col }}\right) \bmod n_{\mathrm{c}} \\
3==>\left(1+2 p_{\text {row }}\right) \bmod n_{\mathrm{r}} & 3===>\left(1+2 p_{\text {col }}\right) \bmod n_{\mathrm{c}} \\
\cdot & \cdot \\
\cdot & \cdot \\
n_{\mathrm{r}}==>\left(1+\left(n_{\mathrm{r}}-1\right) p_{\text {row }}\right) \bmod n_{\mathrm{r}} n_{==}>\left(1+\left(n_{\mathrm{c}}-1\right) p_{\mathrm{col}}\right) \bmod n_{\mathrm{c}}
\end{array}
$$

where $p_{\text {row }}$ and $p_{\text {col }}$ are row-wise and column-wise seeds.

After interleaving in both row-wise and column-wise we get the new location of bits. These new locations are mapped back into $2 \mathrm{D}$ interleavers to get the resulted interleaved bits in $2 \mathrm{D}$.

\subsection{Two Dimensional Random Interleavers}

The concept behind creating two dimensional random interleavers is similar to the concept of prime interleavers. It is also divided into $1 \mathrm{D}$ row-wise and 1D column-wise interleaving. However, it uses random permutation for interleaving instead of using row-wise seed and column-wise seed.

\section{Spreading Codes}

The outcome of a spread system depends greatly upon the spreading codes employed by the transmitters. There are number of spreading codes that can be implemented.

\subsection{Walsh-Hadamard Code}

A set of orthogonal codes which are used as spreading sequences in CDMA systems, is the Walsh-Hadamard codes. Walsh-sequences have the advantage to be orthogonal, in this way we should get rid of any multi-access interference. Although the full-sequence cross-correlation is identically zero, this does not hold for partial-sequence cross-correlation function. The consequence is that the advantage of using orthogonal codes is lost when all users are not synchronized to a single time base. Thus Walsh-sequences are not suitable for some system but they are applied for multi-carrier CDMA and the Cellular CDMA system IS-95 [8].

\subsection{Gold Code}

A Gold code, also known as Gold sequence, is a type of binary sequence which are used in telecommunication (CDMA) [9] and satellite navigation (GPS) [10]. These codes have bounded small cross- 
correlations within a set, which is applicable when multiple devices are broadcasting in the same frequency range. A set of Gold code sequences consists of $2^{\mathrm{n}}-1$ sequences each one with a period of $2^{\mathrm{n}}-1$.

A set of Gold codes $\Gamma$ is given by $c_{x}$ and $c^{x}$ and modulo-2 sums of $c$ and all $M$ different cyclically shifted versions of $c$, hence it contains $M+2$ elements. Another way to number the Gold codes generated by $c$, and $c^{x}$ is: $\Gamma=\left\{c_{0}, c_{1} \ldots \ldots \ldots \ldots \ldots c_{\mathrm{M}}, c_{\mathrm{M}+1}\right\}$

where $c_{0}=c c_{\mathrm{M}+1}=c^{\prime}$ and $c_{\mu}=c+c^{\prime}(\mu),=1, \ldots \ldots \ldots . \mathrm{M}$

Where the sum has to be understood as a modulo-2 sum and $c^{\prime}(\mu)$, is the $m$-sequence given by the

binary representation of $\mu$ as the initial setting for the generating shift register. Concerning the decrease of auto and cross-correlation functions Gold proofed the following proposition: the cross-correlation functions of Gold sequences take only the three values $-1 / M_{3}-t(M), t(M)-2$ where $t(M)$ decreases as $2 / \sqrt{ } M$ for large even $M$ and as $\sqrt{2} / M$ for large odd $M=2 m-1$. To summarize, for large $M$ the peak values of the crosscorrelation functions of Gold codes are much smaller than for the $m$-sequences, but at the cost of higher (but also decreasing) values of the autocorrelation functions. The use of Gold sequences allows the transmission to be asynchronous. The receiver can synchronize using the auto-correlation property of the Gold sequence.

\subsection{Repetition Code Spreading}

In coding theory, the repetition code is one of the most basic error-correcting codes. In order to transmit a message over a noisy channel that may corrupt the transmission in a few places, the idea of the repetition code is to just repeat the message several times [11].

In IDMA, interleavers are the means used for user separation thus the orthogonality condition must be satisfied by these. Repetition spreading in IDMA is used to increase the bandwidth occupancy and hence bandwidth efficiency. The code rate $R_{\mathrm{c}}=1 / L, L$ is the spreading length. In a fading channel Repetition spreading has a diversity gain if the fading amplitudes of the received coded symbols are sufficiently independent.

\subsection{Turbo Code}

It is theoretically possible to approach the Shannon limit by using a block code with large block length or a convolutional code with a large constraint length. The processing power involved to decode such long codes makes this approach impractical.

Turbo codes overcome this limitation by using recursive coders and iterative soft decoders.

The recursive coder forms convolutional codes with short constraint length appear to be block codes with a large block length, and the iterative soft decoder progressively improves the estimate of the received message [12].

Turbo codes are finding use in 3G mobile communications and (deep space) satellite communications as well as other applications where designers seek to achieve reliable information transfer over bandwidth-orlatency-constrained communication link in the presence of data-corrupting noise.

\section{Modulation Techniques In Idma System}

There are a number of digital modulation schemes that have been proposed but there is a trade-off between data rate and bit error rate between the transmitter and receiver [13]. M-ary PSK modulation schemes are one of the most efficient digital data transmission systems as it achieves better bandwidth efficiency than other modulation techniques and give higher data rate [14].

In BPSK scheme, the binary symbols $1 \& 0$ differ only in a relative phase shift of 180 degrees.

Coherent BPSK system is characterized by having a signal space that is one dimensional, with a signal constellation consisting of two message points.

In QPSK scheme, the four symbols are generated for every couple of input bits hence it is known as bandwidth-conserving modulation scheme. Alike BPSK schemes, the QPSK scheme also carries information in terms of the phase variations. In particular, the phase takes on one of four equally spaced values of phase corresponds to a unique pair of message bits. As a result, a QPSK signal has two-dimensional signal constellation and four message points, $M=4$, whose phase angles increase in counter clockwise direction.If special characteristics of QPSK are to be mentioned, we quote that for the same $E_{b} / N_{o}$, a QPSK system transmits the information at twice the bit rate of BPSK for the same channel bandwidth, and QPSK provides twice the spectral efficiency, compared to that of BPSK with the same energy efficiency [15]. 


\section{Comparison Results}

The IDMA scheme, inbuilt with Random Interleaver, imposes the problem of extra bandwidth consumption in the channel, along with high memory requirement at the transmitter and receiver ends. On comparing Bit Error Rate between Random Interleaver, Master Random Interleaver, Tree Based Interleaver and Prime Interleaver we find that these all interleavers have almost same performance. In Fig. 3 However Tree Based Interleaver outperforms the Random Interleavers in terms of memory and bandwidth requirement problems. In terms of computational complexity the Tree Based Interleaver performs better than Master Random Interleaver [5].

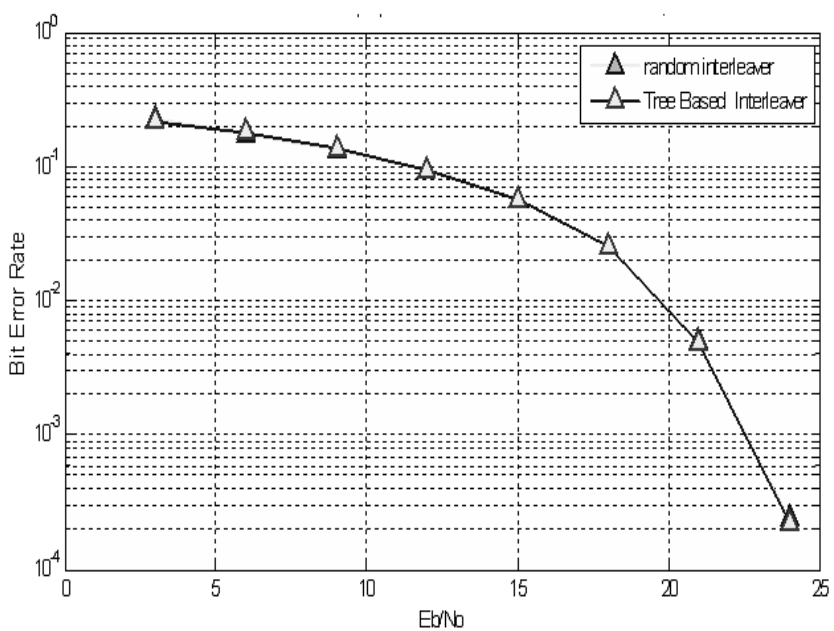

Figure 3. Comparison of Random Interleaver and TBI with 64 users in single path AWGN channel, with uncoded IDMA systems.

\section{Scope For Future}

The testing and improvements in IDMA scheme is being carried out by many researchers throughout the world.IDMA can also be applied in optical networks [16]. In the OR channel of an optical network, the linear summation operation is replaced by non-linear OR operation, which makes it difficult to support uncoordinated multiple access. The IDMA principle provides a solution to this problem while maintaining high optical transmission rates. By refining IDMA detection algorithm we can enhance the performance of IDMA system in wireless communication.

Although IDMA technique is well suited for next generation, but still there are some challenging issues in this scheme such as interleaver design, coding scheme, channel behaviour, optimum signalling scheme etc., in which research is going on in all over the world.

\section{Conclusion}

We have outlined the basic principles of IDMA and have assumed that there are many horizons open for further improvements and testing of IDMA such as in interleaving scheme for memory optimization, improvement in coding schemes, automatic repeat request, synchronization issues, and peak-to-average power reduction, and in modulation schemes. As a relatively new concept, there remain many outstanding issues including pilot signal design, channel estimation, close-loop power control, adaptive transmission and resource allocation.

\section{References}

[1] Pingzhi Fan, "Multiple Access Technologies for Next Generation Mobile Communication",6th International Conference on ITS Telecommunications Proceedings,2006

[2] Ramjee Prasad and Tero O Janepara, "A Survey on CDMA:A Evolution towards Wideband CDMA" in proc. Of IEEE,1998

[3] J. Ch. Fricke, M. Sandell, J. Mietzner, and P. A. Hoeher, "Impact of the Gaussian approximation on the performance of the probabilistic data association MIMO decoder," EURASIPJournal on Wireless Commun. and Networking, vol. 2005, no. 5, pp. 796800, Dec. 2005.

[4] L. Liu, J. Tong, and Li Ping, "Analysis and optimization of CDMA systems with chip-levelinterleavers," IEEE J. Select. Areas Commun. vol. 24, no. 1, pp. 141-150, Jan. 2006

[5] M.Shukla, V.K. Srivastava, S. Tiwari "Analysis and Design of Tree Based Interleaver for Multiuser Receivers in IDMA Scheme," 16th IEEE International Conference on Networks "ICON 2008”, Delhi, India, pp. 1-4, Dec. 13-14, 2008

[6] Yeon, H.C. (n.d.). Performance and bandwidth efficient interleave-division multiple access scheme with high-spread interleavers. In Proceedings of $6^{\text {th }}$ International Conference on Information, Communications \& Signal Processing, Singapore (pp. 1-5).Washington, DC : IEEE. 
[7] M. Shukla, "Performance Analysis of Optimum Interleaver Based on Prime Numbers for Multiuser Iterative IDMA Systems," IGI International Journal of Interdisciplinary Telecommunications and Networking, vol. 2, no. 3, pp. 51-65, 2010.

[8] http://www.wu.ece.ufl.edu/books/EE/communications/CDMA/CDMA.htm

[9] George, M.; Hamid, M.; Miller, A. "Gold Code Generators in Virtex Devices"

[10] "Transmitted GPS Signals". The GPS System. kowoma.de.

[11] http://en.wikipedia.org/wiki/Repetition_code

[12] http://www.philsrockets.org.uk/turbocodes.pdf

[13] Haykin, S.: Digital communication. TMH Publication, Noida (2006)

[14] Chie, C.M.: Bound and approximations for rapid evaluation of coherent MPSK error probabilities. IEEE Trans.Com. 3, 271-273 (1985)

[15] Sanjiv Mishra, V.S. Tripathi, M. Shukla "BPSK and QPSK Modulation Techniques with Optimum Tree Based Interleaver in Iterative IDMA Systems"

[16] H. Chan, M. Griot, A. Vila Casado, R. Wesel, and I. Verbauwhede, "High speed channel coding architectures for the uncoordinated OR channel,’ in Proc. IEEE ASAP’06, PP. 265-268, Colorado, Sept. 11-13, 2006. 